\title{
Intelligent manufacturing and robotics in offshore engineering
}

\begin{abstract}
Intelligent manufacturing and robotics are hot topics all over the world. This paper introduced our researches on the intelligent manufacturing and robotics, such as the largest $2000 \mathrm{~T}$ offshore wind power installation platform in the world.
\end{abstract}

Keywords: intelligent manufacturing, robotics, offshore
Volume 2 Issue 2 - 2018

\author{
Bin $\mathrm{He}$ \\ School of Mechatronic Engineering and Automation, Shanghai \\ University, China
}

\begin{abstract}
Correspondence: Bin He, Shanghai Key Laboratory of Intelligent Manufacturing and Robotics, School of Mechatronic Engineering and Automation, Shanghai University, I 49 Yanchang Road, Shanghai, China, Email mehebin@gmail.com
\end{abstract}

Received: March 12, 2018| Published: April 17, 2018

\section{Introduction}

Intelligent manufacturing and robotics are hot topics all over the world. ${ }^{1}$ This paper discussed the largest $2000 \mathrm{~T}$ offshore wind power installation platform in the world.

\section{T offshore wind power installation platform}

The sea is rich in mineral resources, renewable energy, chemical resources, biological resources and space resources. It is greatly important to provide equipments for the development and utilization of these resources. Offshore engineering equipment is a type of equipments used by humans in the development, use, and protection of offshore activities, which is an important part of strategic emerging industries, intelligent manufacturing industry, and a leading industry for the development of marine economy. It is a prerequisite and basis for the development of marine economy. At present, the development of clean energy has attracted the attention of countries in the world. As a clean renewable energy source, wind energy has the advantages of small turbulence intensity, stable dominant wind direction, saving land resources, stable wind energy, no noise and landscape pollution, abundant resources, and suitable for large-scale development. In recent years, its development and utilization technologies have developed rapidly. At present, European offshore wind power has established a development target of $15 \mathrm{GW}$ in 2030 to supply $14 \%$ of European electricity demand. China has abundant offshore wind power resources and can use more than 700 million kilowatts of offshore wind power with huge potential for development.

The development trend of offshore equipment is oversized tonnage, modularization and intelligence, and it belongs to a small amount or a single piece, the cost is high, and some even do not pass the prototype stage of the prototype, making the production cost and development risk great. It is difficult to ensure the development of offshore equipment through traditional research and development processes and methods. In order to reduce its development risk, improve product quality, and shorten the time to market, virtual prototyping must be adopted. The virtual design, optimization, and simulation of offshore industry equipment through advanced information management tools and the integration of virtual prototyping models of multi-domain systems are necessary to improve the research and development level of offshore industry equipment. The ideal research and development platform needs simple and fast modeling, realistic models, mechanical, hydraulic, and control systems combined to make the mechanical system, power system, and control system work together. This project is developing a 2000T offshore wind power installation platform, which is the largest one in the world. It is a multifunctional combination of multi-fan transportation, dynamic positioning, platform uplifting, large-tonnage lifting fans, piling and accommodation for more workers. Mastering the key core technology of this equipment is of great strategic significance to enhancing the overall strength of designing and manufacturing large-scale marine equipment, promoting the development of marine resources and developing the marine economy.

The offshore wind power installation platform is a comprehensive system engineering integrating many technologies. The overall arrangement design of the offshore wind power installation platform, the design and analysis of crane structure, the design of lifting mechanism and the design and analysis of the pile-pulling machine are all related to the most advanced research and application of related disciplines. Through the research of the 2000T wind power installation platform, this project will design an offshore wind power platform of large tonnage lifting capacity and develop creatively a wind power installation platform with 2000 tons of lifting capacity for the hoisting of $8 \mathrm{MW}$ offshore wind turbine and pile installation.

The platform could cover the standard (offshore 10 kilometers, and water depth more than 10 meters), with 2000T of the crane's maximum lifting capacity, it is an offshore wind power platform with the highest lifting capacity in the world so far. It is $120 \mathrm{~m}$ of the main deck and the maximum height above the water surface during work is up to $160 \mathrm{~m}$. The variable load could reach 10,000 tons when the platform is standing and the available deck area is $3000 \mathrm{~m}^{2}$. It could meet the transportation of 3 sets of $8 \mathrm{MW}$ wind turbines, and the wall thickness of the legs is designed to sections, which reduces the weight of the legs and improves the stability of the towing stability; the X-shaped rack arrangement is used for the first time to reduce the space for lifting equipment and greatly reduce the height of lifting system. So that it reduces the capacity requirements and imbalance of the individual gears. ${ }^{2-3}$ In Figure 1, it shows the structural analysis of spud leg of 2000T offshore wind power installation platform with 
the virtual prototyping technology. Through the project, it could not only overcome technical bottlenecks, master the independent design technology of this world-leading wind power installation platform, but also promote the advancement of science and technology in mechanical and marine specialties and upgrade the scientific and technological strengths in marine heavy industry. It will provide demonstrations for the development of new wind power installation platforms in the future.

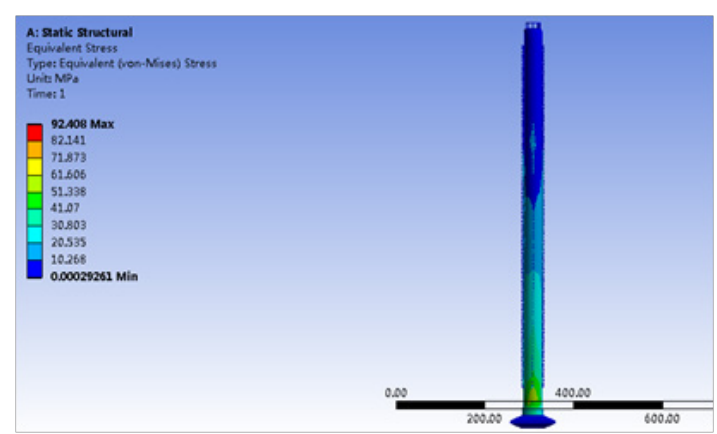

Figure I Structural analysis of spud leg of 2000T offshore wind power installation platform.

\section{Conclusion}

As the intelligent manufacturing and robotics arise the widespread concerns, this paper analyzes the current situation of these topics. And this paper also discussed 2000T offshore wind power installation platform in detail.

\section{Acknowledgement}

The work was supported by Shanghai Science and Technology Commission Project (Grant No. 17DZ1204603).

\section{Conflict of interest}

None.

\section{References}

1. Ministry of Science and Technology of China. "Twelve Five" special plan on intelligent manufacturing technology development. The $12^{\text {th }}$ Beijing International Industrial Automation Exhibition: Beijing; 2012. 3p.

2. He B, Xiao J, Deng Z. Product design evaluation for product environmental footprint. Journal of Cleaner Production. 2018;172:3066-3080.

3. He B, Hua Y. Feature-based integrated product model for low-carbon conceptual design. Journal of Engineering Design. 2017;28(6):408-432. 